\title{
SATB2 Shows Different Profiles Between Appendiceal Adenocarcinomas Ex Goblet Cell Carcinoids and Appendiceal/Colorectal Conventional Adenocarcinomas: An Immunohistochemical Study With Comparison to CDX2
}

\author{
Chen Yang ${ }^{\mathrm{a}, \mathrm{e}}$, Li Sun ${ }^{\mathrm{b}, \mathrm{e}}$, Lingxin Zhang ${ }^{\mathrm{a}}$, Lixin Zhou ${ }^{\mathrm{b}}$, Dongfeng Niu ${ }^{\mathrm{b}}$, Wenfeng $\mathrm{Cao}^{\mathrm{c}}$, \\ Zhongwu Li ${ }^{b}$, Xiaozheng Huang ${ }^{b}$, Qiang Kang ${ }^{b}$, Lin Jia ${ }^{b}$, Marina Platik ${ }^{a}$, \\ Xiuli Liu ${ }^{\mathrm{d}}$, Jinping Lai ${ }^{\mathrm{d}}$, Dengfeng Cao ${ }^{\mathrm{a}, \mathrm{f}}$
}

\begin{abstract}
Background: Special AT-rich sequence-binding protein 2 (SATB2) is a novel marker for colorectal adenocarcinomas but little is known about its expression in appendiceal adenocarcinomas. We aim to investigate SATB2 in these tumors and colorectal adenocarcinomas with comparison to $\mathrm{CDX} 2$.

Methods: Immunohistochemical stains for SATB2 and CDX2 were performed in 49 appendiceal adenocarcinomas (23 conventional, 26 adenocarcinoma ex goblet cell carcinoids (AdexGCCs)) and 57 colorectal adenocarcinomas. Their expression was correlated with tumor differentiation and growth patterns.
\end{abstract}

Results: SATB2 staining was positive in 26/26 (100\%) appendiceal AdexGCCs and 15/23 (65\%) appendiceal conventional adenocarcinomas $(\mathrm{P}=0.001)$. Their mean percentage of SATB2-positive cells was $93 \%$ and $34 \%$, respectively $(\mathrm{P}<0.0001)$. CDX2 staining was seen in 26/26 (100\%) AdexGCCs and 22/23 (96\%) appendiceal conventional adenocarcinomas $(\mathrm{P}=0.4694)$. SATB2 and CDX2 showed similar staining in AdexGCCs but CDX2 labeled more tumor cells than SATB2 in conventional adenocarcinomas (mean $84 \%$ vs. $34 \%, \mathrm{P}$ $<0.0001)$. SATB2 and CDX2 staining was seen in $82 \%(47 / 57)$ and

Manuscript submitted March 31, 2018, accepted April 10, 2018

a Department of Pathology and Immunology, Washington University School of Medicine, Saint Louis, MO 63110, USA

${ }^{b}$ Department of Pathology, Key Laboratory of Carcinogenesis and Translational Research (Ministry of Education), Peking University Cancer Hospital (Beijing Cancer Hospital), Beijing, China

'Department of Pathology, Tianjin Cancer Hospital, Tianjin Medical University, Tianjin, China

${ }^{\mathrm{d}}$ Department of Pathology, Immunology and Laboratory Medicine, University of Florida, Gainesville, FL 32610, USA

${ }^{\mathrm{e}}$ These two authors contributed equally to this project.

${ }^{f}$ Corresponding Author: Dengfeng Cao, Department of Pathology and Immunology, Washington University School of Medicine, 660 S Euclid Ave., Campus Box 8118, St. Louis, MO 63110, USA. Email: dengfengcao@wustl.edu

doi: https://doi.org/10.14740/gr1015w
$96 \%(55 / 57)$ colorectal adenocarcinomas, respectively $(\mathrm{P}=0.01)$. The mean percentage of cells positive for SATB2 and CDX2 was $48 \%$ and $91 \%$, respectively $(\mathrm{P}<0.00001)$. Decreased SATB2 immunoreactivity was associated with non-glandular differentiation particularly signet ring cells in colorectal $(\mathrm{P}=0.001)$ and appendiceal conventional adenocarcinomas $(\mathrm{P}=0.04)$ but not in appendiceal AdexGCCs.

Conclusions: SATB2 is a highly sensitive marker for appendiceal AdexGCCs with similar sensitivity as CDX2. In colorectal and appendiceal conventional adenocarcinomas, SATB2 is not as sensitive as CDX2 and its immunoreactivity is dependent on tumor differentiation.

Keywords: SATB2; CDX2; Adenocarcinoma ex goblet cell carcinoid; Colorectal adenocarcinoma; Appendiceal adenocarcinoma

\section{Introduction}

Special AT-rich sequence-binding protein 2 (SATB2) is a DNA-binding protein involved in transcriptional regulation and chromatin remodeling [1-4]. Studies have shown that SATB2 is a diagnostic marker for osteoblastic differentiation in bone and soft tissue tumors [5] and it is also highly expressed in hindgut well differentiated neuroendocrine tumor [6]. Magnusson et al first showed that SATB2 is a sensitive marker for colorectal adenocarcinoma (sensitivity $85.8 \%$ for primary and $81.3 \%$ for metastasis) with high specificity [7], further confirmed by several other studies [8-12]. Although these studies indicate that SATB2 is a more specific marker than CDX2 for colorectal adenocarcinomas, $15-20 \%$ tumors do not show stain for SATB2 and little is known about the pathologic features associated with negative SATB2 staining [13].

Compared to colorectal adenocarcinomas, information on SATB2 expression in appendiceal adenocarcinomas is very limited [10, 14-15]. Most of the appendiceal tumors investigated were low grade mucinous neoplasms and only two primary appendiceal adenocarcinomas were assessed [10, 14-15]. Appendiceal adenocarcinomas consist of conventional adeno- 
carcinoma (similar to colorectal adenocarcinoma) and adenocarcinoma ex goblet cell carcinoid (AdexGCC) [16]. The former has adenoma as its precursor whereas the latter arises from goblet cell carcinoid (GCC). GCC is also classified as mixed adenoneuroendocrine carcinoma in 2010 WHO classification [16]; however, studies have shown that it is a particular type of adenocarcinoma [17, 18-22]. Tang et al [17] classified GCCs as typical GCCs (type A) and those with invasive adenocarcinomas (type $\mathrm{B}$, with signet ring cell type carcinoma, and type $\mathrm{C}$, with poorly differentiated adenocarcinoma type), though this classification may be somewhat less ideal $[18,21]$. In our recent study, positive SATB2 staining was observed in all 19 $(100 \%)$ metastatic AdexGCCs, indicating SATB2 is a highly sensitive marker for metastatic AdexGCCs [23]. However, the immunohistochemical status of SATB2 in primary appendiceal AdexGCCs and conventional adenocarcinomas is unknown.

To address these questions, we investigated SATB2 immunohistochemical expression in a large series of 57 colorectal adenocarcinomas and 49 appendiceal adenocarcinomas (23 conventional, 26 AdexGCCs). We correlated SATB2 immunoreactivity with tumor differentiation and growth patterns in these tumors. We also compared SATB2 to CDX2 for their sensitivity.

\section{Materials and Methods}

\section{Materials}

This study was approved by the Institutional Review Board. The surgical pathology archive of Washington University was searched for colorectal/appendiceal adenocarcinomas with or without signet ring cells, pure signet ring cell carcinomas and appendiceal AdexGCCs. Fifty-seven in-house colorectal adenocarcinomas (2011 to 2015) and 27 appendiceal adenocarcinomas (1990 to 2017) were collected. Hematoxylin and eosin slides were re-reviewed to confirm the diagnosis. Additional appendiceal adenocarcinomas were retrieved from Peking University Cancer Hospital (Beijing, China) ( $\mathrm{N}=11,2011$ to 2014), Tianjin Cancer Hospital (Tianjin, China) $(\mathrm{N}=9,2011$ to 2016) and University of Florida $(\mathrm{N}=2,2017)$.

These 57 colorectal carcinomas were well differentiated (WD) in 11, moderately differentiated (MD) in 19 and poorly differentiated (PD) in 27. Among them, nine were pure (100\%) glandular WD adenocarcinomas, 10 pure signet ring cell carcinomas (100\% signet ring cells), and 38 adenocarcinomas with variable percentage of glandular, non-glandular non-signet ring cells and/or signet ring cells. Among 23 conventional type appendiceal adenocarcinomas (seven WD, eight MD, eight PD), four showed pure glandular growth, one was pure signet ring cell carcinoma, and 18 had mixed components (12 with mixed glandular and non-glandular non-signet ring cell components, one with mixed signet ring cell and non-glandular non-signet ring cell component, five with all three components). The appendiceal AdexGCCs typically show multiple growth patterns including typical cryptal pattern, poorly cohesive goblet/signet ring cells and non-mucinous cells, microglandular/tubular glands and solid growth etc as described previously [16-17, 20-21]. All 26 appendiceal AdexGCCs have at least focal background residual GCC.

\section{Immunohistochemical staining}

One to two representative formalin fixed paraffin tissue blocks containing tumor from each case were retrieved to generate $4 \mu \mathrm{m}$ thick unstained slides for immunohistochemical staining for SATB2 (dilution 1:100, clone EPNCIR 130A, Abcam, Cambridge, MA) and CDX2 (prediluted, clone EPR2764Y, Cell Marque, Rocklin, CA) on a Ventana Benchmark automated immunostainer (Ventana Medical Systems, Inc., Tucson, AZ) according to standard protocols with appropriate positive and negative controls. Chromogranin (prediluted, clone LK2H10, Ventana, Tucson, AZ) and synaptophysin (predilute, polyclonal, Cell Marque, Rocklin, CA) were also performed for AdexGCCs using a Ventana Benchmark automated immunostainer.

Only nuclear staining was considered positive for SATB2 and CDX2. The staining was reviewed in a consensus manner (CY, LZ, DC) with a multiheaded microscope. The results were scored in a semi-quantitative pattern as $0(<1 \%$ cells $), 1+(1-$ $25 \%), 2+(26-50 \%), 3+(51-75 \%), 4+(76-100 \%)$. The percentage of positive cells was visually estimated with $5 \%$ increment.

\section{Statistical analysis}

The Fisher exact test was used to compare percentage of cases with positive staining and the staining patterns. The paired and non-paired $t$-tests were used to compare the mean percentage of tumor cells stained with SATB2 and CDX2. P-value less than 0.05 was considered statistically significant.

\section{Results}

\section{SATB2 expression in appendiceal adenocarcinomas ex goblet cell carcinoids $(\mathrm{N}=26)$}

All 26 (100\%) AdexGCCs showed positive SATB2 staining including $2+$ in one $(4 \%)(50 \%$ cells), $3+$ in one $(4 \%)(60 \%$ cells), and $4+$ in $24(92 \%)$. There is no SATB2 staining difference between AdexGCCs and background residual GCCs (Fig. $1,2)$. The mean percentage of SATB2-positive tumor cells in AdexGCCs was 93\% (median 95\%, range 50-100\%). SATB2 stained at least 90\% tumor cells in 24/26 (92\%) AdexGCCs. Different growth patterns in AdexGCCs showed similar SATB2 staining (Fig. 2).

\section{SATB2 expression in appendiceal conventional type adeno- carcinomas $(\mathbf{N}=\mathbf{2 3})$}

Positive SATB2 staining was seen in 15/23 (65\%) appendiceal conventional adenocarcinomas, including in $6 / 7$ (86\%) WD, 4/8 (50\%) MD, and 5/8 (63\%) PD (Table 1, Fig. 3). The mean percentage of SATB2-positive tumor cells was $52 \%$ for WD, $25 \%$ for $\mathrm{MD}$, and $27 \%$ for $\mathrm{PD}$ adenocarcinomas, respectively $(\mathrm{P}>$ 


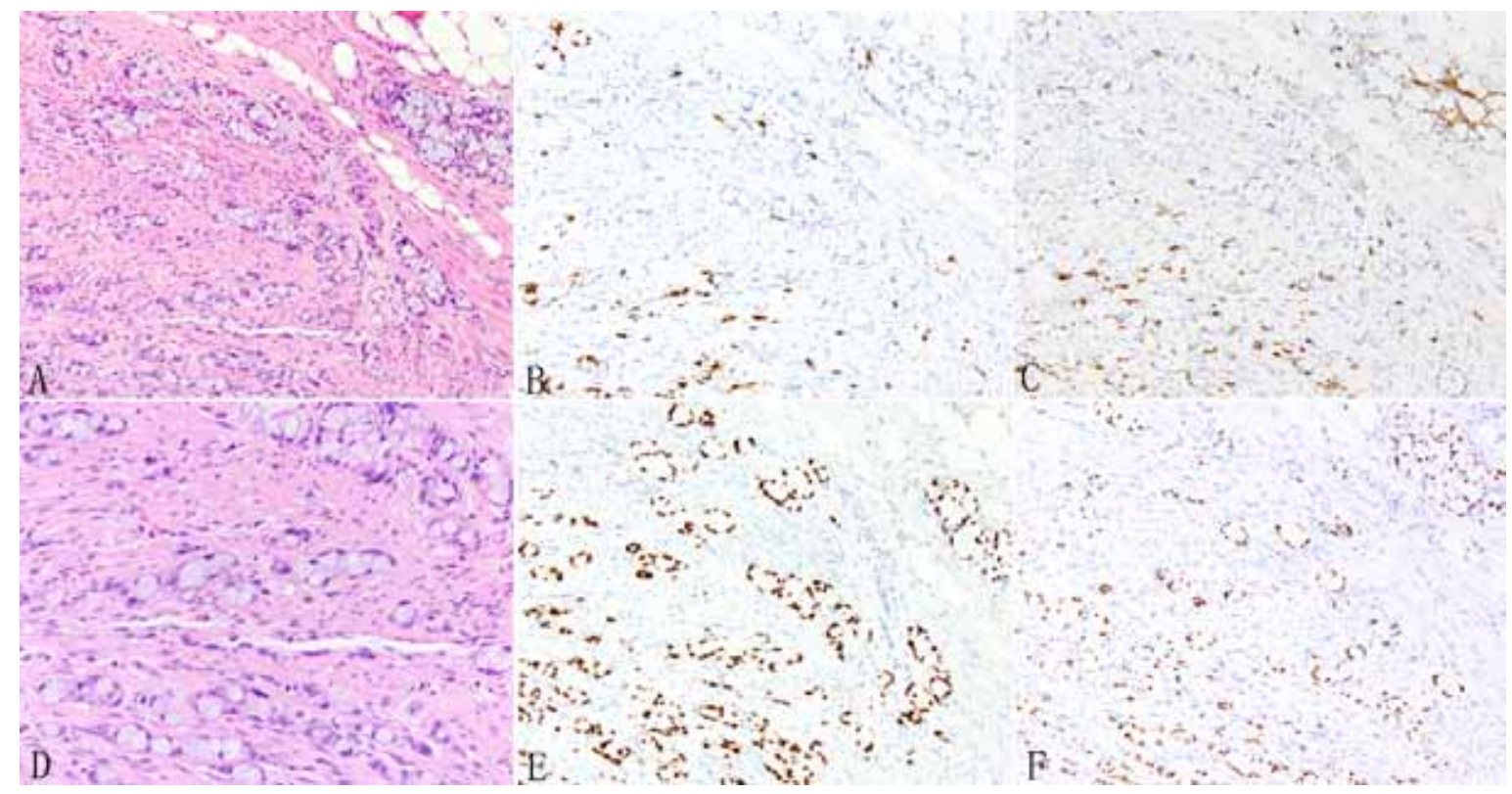

Figure 1. Expression of SATB2 in appendiceal goblet cell carcinoids. Goblet cell carcinoid is morphologically characterized by well-defined goblet cells in clusters or cohesive linear fashion infiltrating appendiceal wall and peri-appendiceal soft tissue (A, D). The tumor cells often show focal chromogranin (B) and synaptophysin (C) staining but diffuse staining for CDX2 (E) and SATB2 $(\mathrm{F})$.

0.05 between any two). Among the seven cases with a signet ring cell component, only three $(43 \%)$ were positive for SATB2 staining (mean positive cells: $11 \%$; median 0 , range $0-45 \%$ ).

At the individual component/growth pattern level, the mean percentage of SATB2-positive tumor cells was 35\% (median $10 \%$, range $0-95 \%$ ) in the 19 glandular components, $18 \%$ (median 3\%, range $0-95 \%$ ) in the 18 non-glandular non- signet ring cell components, and $7 \%$ (median $0 \%$, range $0-30 \%$ ) in the seven signet ring cell components $(\mathrm{P}=0.02$ for glandular vs. non-glandular, $\mathrm{P}=0.06$ for glandular vs. non-signet nonglandular, $\mathrm{P}=0.03$ for glandular vs. signet ring cell).

\section{SATB2 expression in colorectal adenocarcinomas $(N=57)$}

Positive SATB2 staining was seen in 47/57 (82\%) colorectal adenocarcinomas, including in $11 / 11$ (100\%) WD, $15 / 19$ (79\%) MD, and 21/27 (78\%) PD adenocarcinomas (semiquantitative staining pattern $/ 0$ to $4+: \mathrm{P}=0.009$ for $\mathrm{WD}$ vs. $\mathrm{MD}$ vs. $\mathrm{PD}, \mathrm{P}=0.1218$ for $\mathrm{WD}$ vs. $\mathrm{MD}, \mathrm{P}=0.001$ for $\mathrm{WD}$ vs. $\mathrm{PD}$, $\mathrm{P}=0.073$ for $\mathrm{MD}$ vs. $\mathrm{PD}, \mathrm{P}=0.007$ for $\mathrm{WD}$ vs. $\mathrm{MD}+\mathrm{PD}, \mathrm{P}=$ 0.002 for low grade vs. high grade) (Table 2, Fig. 4). The mean percentage of SATB2-positive cells was $84 \%$ for WD, $53 \%$ for $\mathrm{MD}$, and $29 \%$ for $\mathrm{PD}$ adenocarcinomas, respectively $(\mathrm{P}<$ 0.01 between any two). Among the 37 adenocarcinomas with a signet ring cell component, positive SATB2 staining was seen in $29(78 \%)$ cases, including $11 / 13(85 \%)$ cases with $1-50 \%$ signet ring cells, $12 / 14(86 \%)$ cases with $50-95 \%$ signet ring cells, and $6 / 10(60 \% ; 2+$ in four, $3+$ in one, $4+$ in one) pure signet ring cell carcinomas.

At the tumor component/growth pattern level, the mean percentage of SATB2-positive tumor in the 37 signet ring cell components was $24 \%$ (median $10 \%$, range $0-95 \%$ ), significantly lower than $63 \%$ (median $80 \%$, range $0-100 \%$ ) in the 36 glandular components and 49\% (median 50\%, range $0-95 \%$ ) in the 29 non-glandular non- signet ring cell components $(\mathrm{P}=$ $0.01)$. The glandular components had a higher percentage of SATB2-positive tumor cells than the non-glandular non-signet ring cell components, but their difference did not reach statistical significance $(63 \%$ vs. $49 \%, \mathrm{P}=0.09)$. The glandular component in those with a pure glandular growth pattern $(\mathrm{N}=$ 9) showed more SATB2 staining than that in those also with other growth patterns $(\mathrm{N}=27)(83 \%$ vs. $56 \%, \mathrm{P}=0.06)$. The signet ring cell component showed similar SATB2 staining between those with mixed signet ring cells and other component (s) and those pure signet ring cell carcinomas (23\% vs. $29 \%$, $\mathrm{P}>0.05)$.

\section{Comparison of SATB2 staining between appendiceal ad- enocarcinomas ex goblet cell carcinomas and appendiceal/ colorectal conventional adenocarcinomas}

Appendiceal AdexGCCs $(\mathrm{N}=26)$ showed significantly more SATB2 staining than conventional adenocarcinomas $(\mathrm{N}=23)$ (percentage of cases positive for SATB2: $100 \%$ vs. $65 \%, \mathrm{P}=$ $0.001 ; \mathrm{P}=5.0 \mathrm{E}-06$ for semi-quantitative staining pattern $(0$ to $4+$ ); mean positive tumor cells $93 \%$ vs. $34 \%, \mathrm{P}<0.0001$ ) (Table 3).

Appendiceal AdexGCCs also showed significantly more SATB2 staining than colorectal adenocarcinomas (percentage of cases positive for SATB2: $100 \%$ vs. $82 \%, P=0.03 ; \mathrm{P}=0.004$ for semi-quantitative staining pattern ( 0 to $4+$ ); $\mathrm{P}<0.0001$ for mean positive tumor cells, $93 \%$ vs. $48 \%$ ) (Table 3 ). 


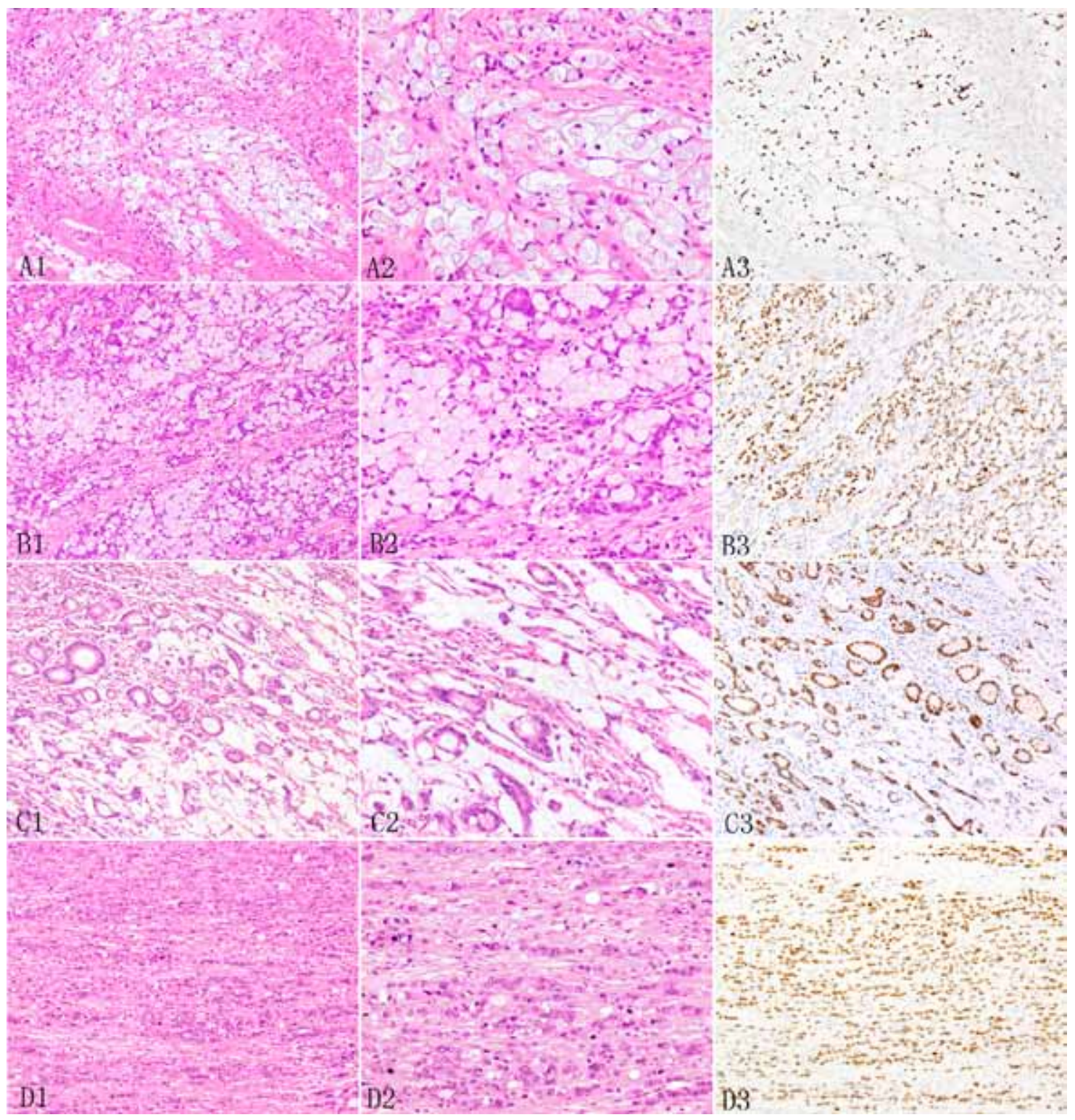

Figure 2. Expression of SATB2 in appendiceal adenocarcinoma ex goblet cell carcinoid. The signet ring cell type may show desmoplastic stroma and extracellular mucin pools (A1, A2) and irregular large nests and sheet (B1, B2). In the poorly differentiated adenocarcinoma type, the invasive adenocarcinoma may manifest as tubular glands $(C 1, C 2)$ or individual to clusters to small nested undifferentiated cells with focal small glandular lumen (D1, D2). The invasive adenocarcinoma cells show diffuse SATB2 staining (A3, B3, C3, D3).

\section{Comparison of SATB2 to CDX2 in appendiceal adenocar- cinomas ex goblet cell carcinoids, appendiceal convention- al adenocarcinomas and colorectal adenocarcinomas}

All $26(100 \%)$ appendiceal AdxeGCCs showed strong 4+ CDX2 staining (mean 98\% positive cells, range 95-100\%). There is no significant difference between SATB2 and CDX2 in the semi-quantitative staining pattern $(\mathrm{P}=0.3534)$. Both SATB2 and CDX2 showed $100 \%$ sensitivity for AdexGCCs (P $=1.0$ ), however, $\mathrm{CDX} 2$ stained more tumor cells than SATB2 (mean $98 \%$ vs. $93 \%, \mathrm{P}=0.02$ ) (Table 3 ).
Positive CDX2 staining was seen in 22/23 (96\%) appendiceal conventional adenocarcinomas but only 15 of $23(65 \%)$ were positive for SATB2 $(\mathrm{P}=0.01$, Tables 1,3$)$. CDX2 showed similar staining among different degree of differentiation (Table 2) and among different histologic patterns (mean positive cells: $86 \%$ for glandular component, $82 \%$ for signet ring cell component, $72 \%$ for non-glandular non-signet ring cell component, $\mathrm{P}>0.05$ ). The number of mean SATB2-positive tumor cells in these tumors was significantly lower than that for CDX2 (34\% vs. $84 \%, \mathrm{P}=1.2 \mathrm{E}-05)$ (Table 3 ).

Among the 57 colorectal adenocarcinomas, 56 (98\%) showed positive CDX2 staining, significantly higher than that 


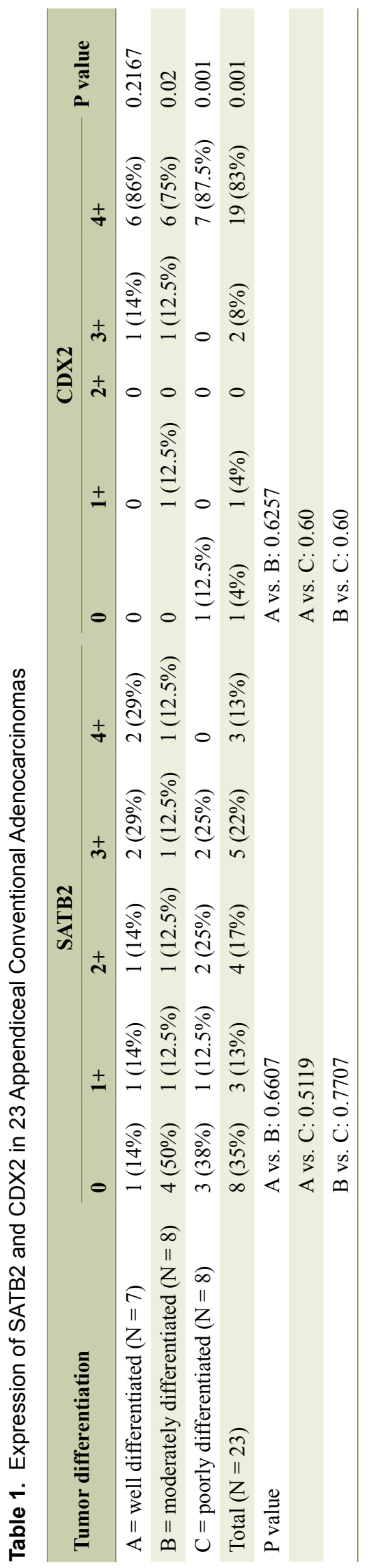

for SATB2 (47/57 or 82\%) $(\mathrm{P}=0.004)$ (Tables 2,3$)$. SATB2 and CDX2 showed similar semi-quantitative staining pattern in 11 WD tumors $(\mathrm{P}=0.5901)$ but $\mathrm{CDX} 2$ demonstrated significantly more semi-quantitative staining than SATB2 in $19 \mathrm{MD}$ and $27 \mathrm{PD}$ tumors $(\mathrm{P}<0.05$, Table 2$)$. The mean percentage of positive tumor cells for SATB2 and CDX2 was $84 \%$ and $95 \%$ for WD adenocarcinomas $(\mathrm{P}=0.04), 53 \%$ and $90 \%$ for MD ( $\mathrm{P}=0.006), 29 \%$ and $90 \%$ for $\mathrm{PD}$ adenocarcinomas $(\mathrm{P}$ $<0.00001$ ), respectively. Different histologic patterns showed similar CDX2 staining (mean positive tumor cells: 91\% in the glandular component, $95 \%$ in the signet cell component, $82 \%$ in the non-glandular non-signet ring cell component, $\mathrm{P}>0.05$ between any two).

\section{Discussion}

In this study, we investigated SATB2 immunoreactivity in 26 appendiceal AdexGCCs and 80 conventional appendiceal/ colorectal adenocarcinomas. Consistently high expression of SATB2 was seen in all histologic patterns including signet ring cells in all $26(100 \%)$ AdexGCCs. However, only $65 \%(15 / 23)$ conventional appendiceal and $82 \%$ (47/57) colorectal adenocarcinomas showed SATB2 immunoreactivity.

Our findings indicate SATB2 is a highly sensitive diagnostic marker for primary appendiceal AdexGCCs. We previously showed similar SATB2 sensitivity for metastatic appendiceal AdexGCCs (19/19 or $100 \%)$ [23]. The percentage of tumor cells with SATB2 immunoreactivity is high in both primary and metastatic AdexGCCs (average 93\% in primary, 97\% in metastasis) [23]. Prior to our study, there has been only one study on SATB2 in six high grade appendiceal adenocarcinomas (two primary cases, four metastatic cases) [10]. All these six tumors were positive for SATB2 but the authors did not specify the subtypes and the extent of positivity [10]. Two other studies showed positive SATB2 staining in 63/72 appendiceal mucinous neoplasms (extent of positivity unknown) but it is unknown how many of these mucinous tumors are mucinous adenocarcinomas [14-15].

GCCs (with/without associated adenocarcinomas) often present with advanced stages and involve adjacent right colon and ileum [16-17, 20-21]. In two large series, 78 of 110 (71\%) appendiceal GCCs presented with T4 disease $[17,20]$. Reid et al showed that $58 \%(33 / 57)$ female patients had disseminated gynecologic tract disease (mainly in the ovary) [21]. Due to the diffuse involvement of appendix and adjacent colon and abdominopelvic cavity, it is often difficult to determine whether a poorly differentiated adenocarcinoma with signet ring cells involving these organs is an appendiceal AdexGCC or a conventional appendiceal/colorectal adenocarcinoma with signet ring cells. Although these two types of adenocarcinomas are surgically managed similarly, there is some prognostic difference $[17,20]$. Given their overlapping morphologic features, pathologists often rely on immunohistochemical markers to facilitate the differential diagnosis. However, appendiceal AdexGCC and appendiceal/colorectal conventional PD adenocarcinoma show similar CDX2 and CK20 profiles [23-26] as well as overlapping CK7 profile 


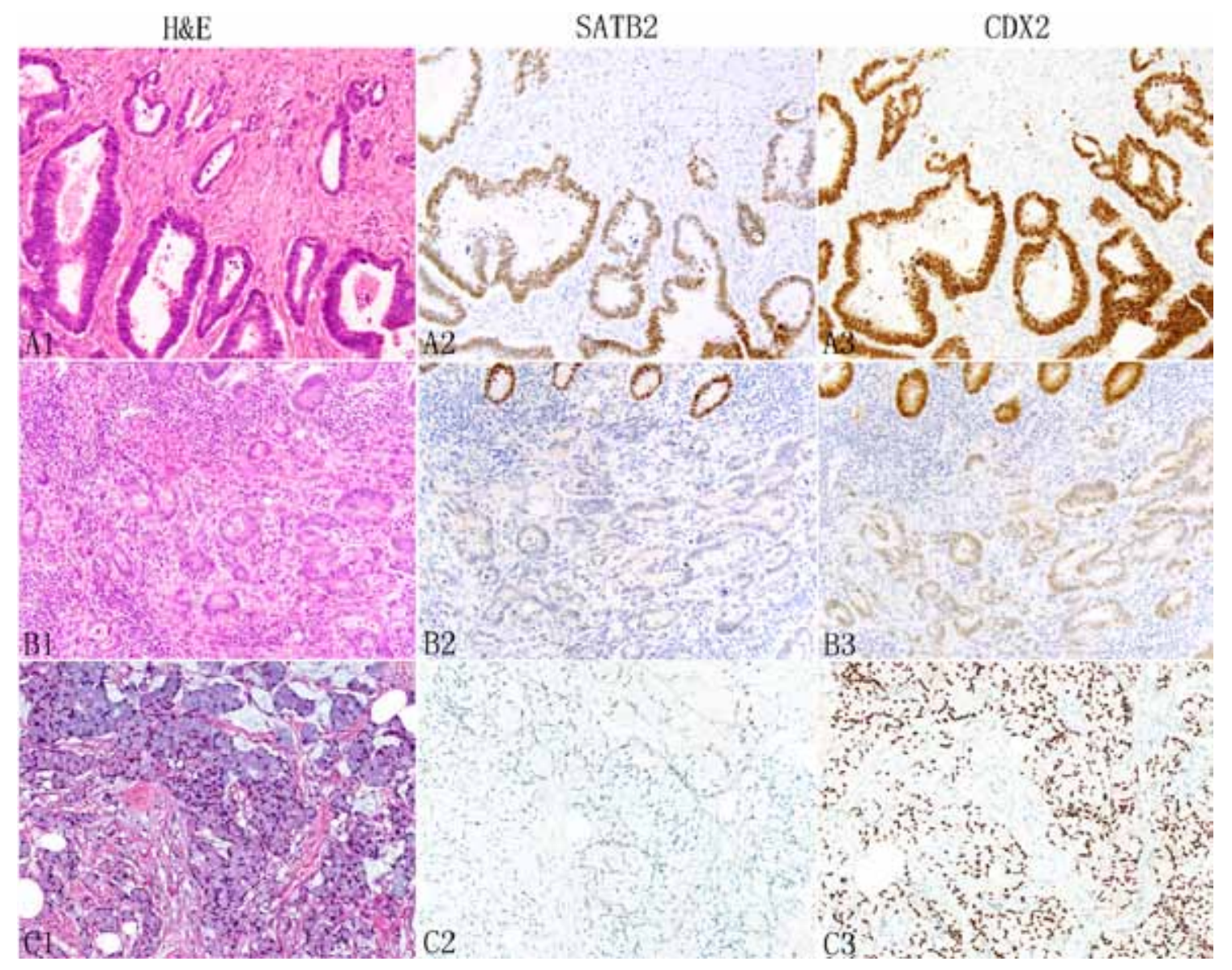

Figure 3. Expression of SATB2 and CDX2 in appendiceal conventional adenocarcinomas. The neoplastic glands typically show more SATB2 staining than non-glandular components such as individual tumor cells (A1: H\&E, A2: SATB2). In some tumors, both glandular and non-glandular components may lose SATB2 expression (B1: moderately differentiated adenocarcinoma; B2: SATB2, top shows normal glands with strong SATB2 staining). Signet ring cells (C1) are also associated with decreased SATB2 staining (C2). In contrast, CDX2 typically shows diffuse strong staining (A3 and C3) with occasional focal weak staining (B3).

(in $60 \%$ AdexGCCs, in $35-40 \%$ of PD and mucinous colorectal adenocarcinomas, and $17 \%$ colorectal signet ring cell carcinomas) [24-28]. Although GCC is considered a mixed adenoneuroendocrine carcinoma, chromogranin and synaptophysin are often under-expressed, especially those with AdexGCCs. Hristov et al showed chromogranin and synaptophysin staining in $38 \%$ and $40 \%$ appendiceal GCCs with AdexGCCs, respectively [19]. Although there is no data on chromogranin and synaptophysin in primary colorectal adenocarcinomas with signet ring cells, we recently showed that metastatic colorectal PD carcinomas with signet ring cells in the ovary showed positive chromogranin and synaptophysin in $31 \%$ and $62 \%$ cases, respectively [23]. Therefore these common markers including CDX2, CK7, CK20, chromogranin and synaptophysin lack adequate sensitivity and specificity to distinguish appendiceal AdexGCCs from conventional appendiceal/colorectal PD carcinomas with signet ring cells. Our current study indicates that SATB2 may have some diagnostic utility in some cases to distinguish appendiceal AdexGCCs from colorectal/appendiceal conventional adenocarcinomas with signet ring cells. Given the $100 \%$ sensitivity of SATB2 for appendiceal AdexGCCs, a poorly differentiated carcinoma with signet ring cells involving appendix and adjacent colon showing negative SATB2 staining is unlikely to be an appendiceal AdexGCCs (negative predictive value $100 \%$ ). In our study, $22 \%$ colorectal and $57 \%$ appendiceal conventional adenocarcinomas with a signet ring cell component were negative for SATB2 staining.

Distinguishing appendiceal AdexGCCs from conventional PD appendiceal/colorectal adenocarcinomas with signet ring cell not only has prognostic significance but also may affect the patients' follow-up schedule and non-surgical treatment options. Appendiceal AdexGCCs only seldom show liver (4\%) and lung $(1 \%)$ metastasis despite their widespread growth in the abdominopelvic cavity [21]. Although this pattern is similar to appendiceal mucinous adenocarcinomas, it differs from PD colorectal adenocarcinomas with signet ring cells. Conventional colorectal adenocarcinomas eventually develop liver and lung metastasis in 50\% and 10-20\% cases, respectively [29-30]. In addition, given the tendency of appendiceal AdexGCCs to recur in the peritoneal and pelvic space, these patients may benefit from additional intraoperative hyperther- 


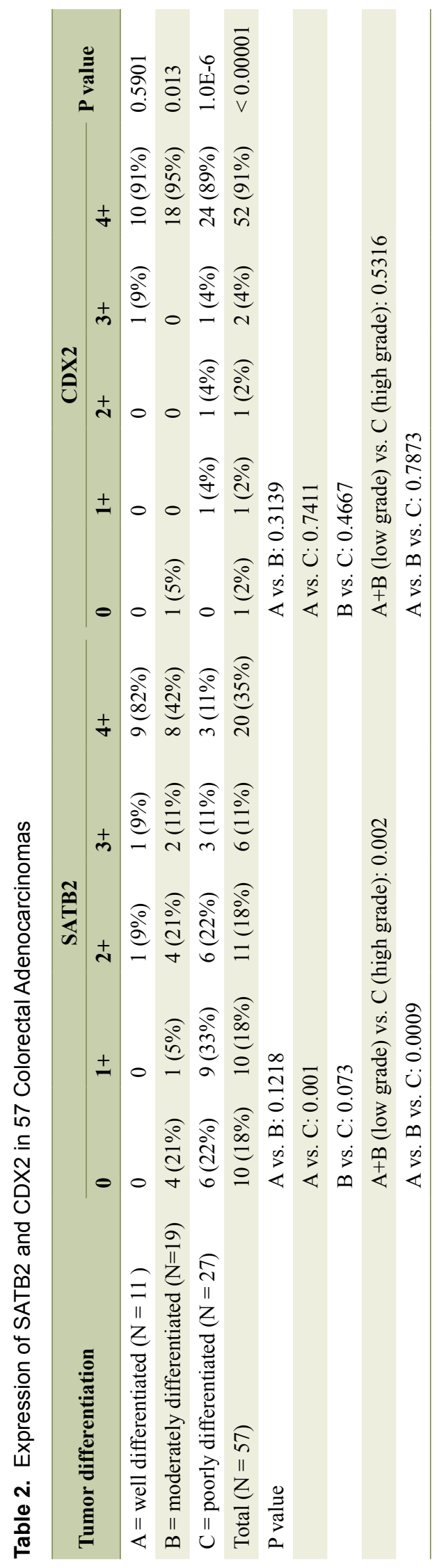

mic chemotherapy as used for disseminated appendiceal mucinous adenocarcinomas [31].

In this study, we also compared SATB2 to CDX2 for their sensitivity. We found that SATB2 was not as sensitive as CDX2 for conventional appendiceal and colorectal adenocarcinomas (Tables 1-3). The reported average sensitivity of SATB2 and CDX2 for colorectal adenocarcinomas was $84.4 \%(60 \%$ to $97 \%)(\mathrm{N}=2028)$ and $97 \%(95 \%$ to $100 \%)$ $(\mathrm{N}=508)$, respectively $(\mathrm{P}<0.0001$, Fisher exact test) [5-10]. Our results were in keeping with those from these prior studies [5-10]. However, our study differed from prior studies in that we correlated SATB2 immunoreactivity with tumor differentiation/growth pattern and delineated the histologic patterns associated with decreased SATB2 immunoreactivity. Previously Magnusson et al briefly mentioned that PD colorectal adenocarcinomas showed an apparently less amount of staining than WD ones but they did not elaborate further [7]. We found SATB2 immunoreactivity in appendiceal/colorectal conventional adenocarcinomas was associated with tumor differentiation, and presence of non-glandular differentiation, particularly signet ring cells, is associated with decreased immunoreactivity. This feature of SATB2 has diagnostic implications. Negative SATB2 staining in a metastatic moderately to poorly differentiated adenocarcinoma does not exclude a colorectal origin as $21 \%$ such primary tumors have negative SATB2 staining. In addition, colorectal adenocarcinomas may lose SATB2 immunoreactivity during metastases (12\% tumors in one prior study) [7]. Therefore using only SATB2 to confirm or exclude metastatic colonic adenocarcinoma will miss a significant percentage of cases $(20 \%)$. In contrast, CDX2 demonstrates consistently high expression in colorectal adenocarcinomas of various differentiations/ growth patterns and their metastasis [7-13]. However, CDX2 is less specific than SATB2 for colorectal adenocarcinoma [8-12, 32]. For example, 29\% esophageal and $47 \%$ gastric adenocarcinomas are also positive for CDX2 [32] but they are only rarely positive for SATB2 [8]. Gastroesophageal adenocarcinoma is often in the differential diagnosis for metastatic colorectal adenocarcinoma. Therefore, when metastatic colorectal adenocarcinoma is in the differential diagnosis, both SATB2 and CDX2 should be included to increase the sensitivity and specificity.

Although AdexGCCs and conventional colorectal/appendiceal adenocarcinomas show morphologic overlapping, they have different genetic profiles. Studies have shown that frequently mutated genes in the colorectal adenocarcinomas including TP53, KRAS and $A P C$ were not or rarely mutated in AdexGCCs [33-34]. In contrast, AdexGCCs harbored mutations in Wnt-signaling-associated genes including USP9X, NOTCH1, CTNNA1, CTNNB1 and TRRAP [34]. Appendiceal conventional and colorectal adenocarcinomas showed similar genetic mutations [33]. Different SATB2 immunohistochemical profiles between appendiceal AdexGCCs and appendiceal/colorectal conventional adenocarcinomas add additional evidence that AdexGCCs are distinct tumors. AdexGCC and GCC are thought to arise from pluripotent intestinal epithelial crypt base stem cells (crypt cell carcinoma) [22, 26].

One limitation in our study is that the number of appendiceal conventional adenocarcinomas is relatively small as these 


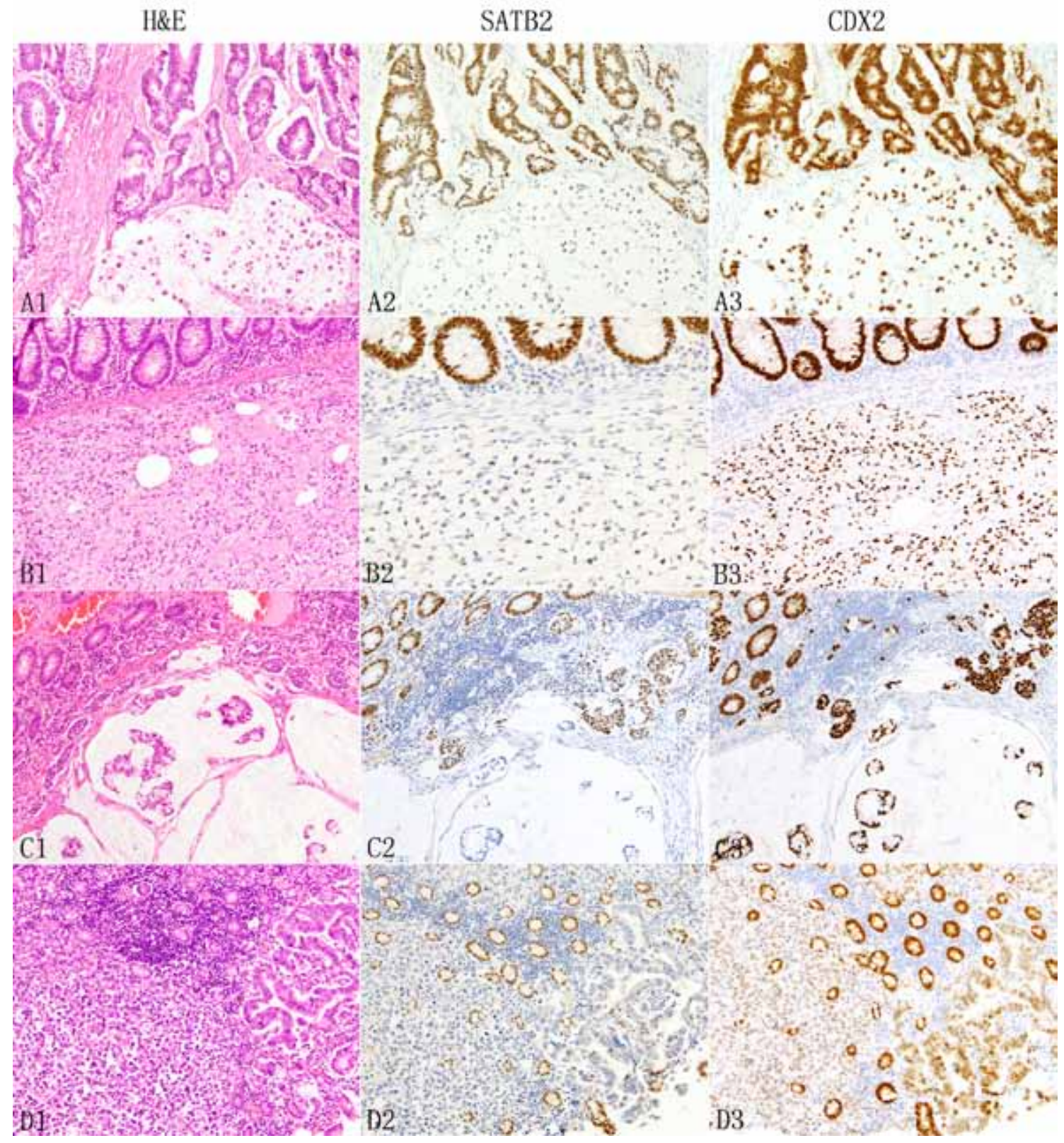

Figure 4. Expression of SATB2 and CDX2 in colorectal adenocarcinomas. In colorectal adenocarcinomas, the neoplastic glands (A1 top) typically show diffuse strong SATB2 staining (A2) but signet ring cells, either admixed with glands (A1 bottom) or pure (B1) are associated with absent (A2 bottom) or decreased/weak (B2) SATB2 immunoreactivity. The presence of colloid carcinoma component ( $\mathrm{C} 1$ bottom) is often associated with decreased or absent SATB2 staining (C2 botttom). Non-glandular non-signet ring cell component such as single cells, clusters and nests (C1, D1) are also associated with decreased (C2 middle) or absent (D2) SATB2 immunoreactivity. In D1, the neoplastic glands next to the discohesive tumor cells are also negative for SATB2 staining (D2). The background benign colonic glands show strong diffuse SATB2 staining (B2 to D2). In contrast, CDX2 demonstrates consistently diffuse staining across different growth patterns, mostly strong (A3 to C3) with occasional weak (D3) staining.

tumors are relatively uncommon [16].

\section{Conclusions}

In summary, SATB2 shows different immunohistochemical profiles between AdexGCCs and colorectal/appendiceal con- ventional adenocarcinomas. SATB2 demonstrates consistently high expression in AdexGCCs but its immunoreactivity in colorectal/appendiceal conventional adenocarcinomas is associated with tumor growth pattern and differentiation. SATB2 and CDX2 show similar sensitivity for AdexGCCs but SATB2 is not as sensitive as CDX2 for colorectal/appendiceal conventional adenocarcinomas. Our study is the first one to investi- 
gate SATB2 in primary appendiceal AdexGCCs and delineate the histologic patterns associated with deceased SATB2 immunoreactivity in colorectal/appendiceal conventional adenocarcinomas.

\section{Acknowledgments}

The authors want to thank Matthew Zipprich for help with immunohistochemical staining.

\section{Conflict of Interest}

The authors declare no conflict of interest.

\section{References}

1. Dobreva G, Chahrour M, Dautzenberg M, Chirivella L, Kanzler B, Farinas I, Karsenty G, et al. SATB2 is a multifunctional determinant of craniofacial patterning and osteoblast differentiation. Cell. 2006;125(5):971-986.

2. Britanova O, Depew MJ, Schwark M, Thomas BL, Miletich I, Sharpe P, Tarabykin V. Satb2 haploinsufficiency phenocopies 2q32-q33 deletions, whereas loss suggests a fundamental role in the coordination of jaw development. Am J Hum Genet. 2006;79(4):668-678.

3. Britanova O, de Juan Romero C, Cheung A, Kwan KY, Schwark M, Gyorgy A, Vogel T, et al. Satb2 is a postmitotic determinant for upper-layer neuron specification in the neocortex. Neuron. 2008;57(3):378-392.

4. Alcamo EA, Chirivella L, Dautzenberg M, Dobreva G, Farinas I, Grosschedl R, McConnell SK. Satb2 regulates callosal projection neuron identity in the developing cerebral cortex. Neuron. 2008;57(3):364-377.

5. Conner JR, Hornick JL. SATB2 is a novel marker of osteoblastic differentiation in bone and soft tissue tumours. Histopathology. 2013;63(1):36-49.

6. Li Z, Yuan J, Wei L, Zhou L, Mei K, Yue J, Gao H, et al. SATB2 is a sensitive marker for lower gastrointestinal well-differentiated neuroendocrine tumors. Int J Clin Exp Pathol. 2015;8(6):7072-7082.

7. Magnusson K, de Wit M, Brennan DJ, Johnson LB, McGee SF, Lundberg E, Naicker K, et al. SATB2 in combination with cytokeratin 20 identifies over $95 \%$ of all colorectal carcinomas. Am J Surg Pathol. 2011;35(7):937-948.

8. Lin F, Shi J, Zhu S, Chen Z, Li A, Chen T, Wang HL, et al. Cadherin-17 and SATB2 are sensitive and specific immunomarkers for medullary carcinoma of the large intestine. Arch Pathol Lab Med. 2014;138(8):1015-1026.

9. Dragomir A, de Wit M, Johansson C, Uhlen M, Ponten F. The role of SATB2 as a diagnostic marker for tumors of colorectal origin: Results of a pathology-based clinical prospective study. Am J Clin Pathol. 2014;141(5):630638.

10. Moh M, Krings G, Ates D, Aysal A, Kim GE, Rabban JT. SATB2 expression distinguishes ovarian metastases of colorectal and appendiceal origin from primary ovar- 
ian tumors of mucinous or endometrioid type. Am J Surg Pathol. 2016;40(3):419-432.

11. Brandler TC, Jelloul FZ, Soto D, Das K, Rosen L, Bhuiya TA. Young investigator challenge: Cadherin-17 and SATB2 in cytology specimens: Do these new immunostains help in differentiating metastatic colorectal adenocarcinoma from adenocarcinomas of other origins? Cancer Cytopathol. 2015;123(12):706-713.

12. Perez Montiel D, Arispe Angulo K, Cantu-de Leon D, Bornstein Quevedo L, Chanona Vilchis J, Herrera Montalvo L. The value of SATB2 in the differential diagnosis of intestinal-type mucinous tumors of the ovary: primary vs metastatic. Ann Diagn Pathol. 2015;19(4):249-252.

13. Berg KB, Schaeffer DF. SATB2 as an Immunohistochemical marker for colorectal adenocarcinoma: a concise review of benefits and pitfalls. Arch Pathol Lab Med. 2017;141(10):1428-1433.

14. Li Z, Roth R, Rock JB, Lehman A, Marsh WL, Suarez A, Frankel WL. Dual immunostain with SATB2 and CK20 differentiates appendiceal mucinous neoplasms from ovarian mucinous neoplasms. Am J Clin Pathol. 2017;147(5):484-491.

15. Strickland S, Parra-Herran C. Immunohistochemical characterization of appendiceal mucinous neoplasms and the value of special AT-rich sequence-binding protein 2 in their distinction from primary ovarian mucinous tumours. Histopathology. 2016;68(7):977-987.

16. Lauwers G. In: Bosman FT, Carneiro F, Hruban RH, et al., eds. WHO classification of tumors of the digestive system. 4th ed. Lyon: IARC Press; 2010. p. 48-58.

17. Tang LH, Shia J, Soslow RA, Dhall D, Wong WD, O’Reilly E, Qin J, et al. Pathologic classification and clinical behavior of the spectrum of goblet cell carcinoid tumors of the appendix. Am J Surg Pathol. 2008;32(10):1429-1443.

18. Wang HL, Dhall D. Goblet or signet ring cells: that is the question. Adv Anat Pathol. 2009;16(4):247-254.

19. Hristov AC, Young RH, Vang R, Yemelyanova AV, Seidman JD, Ronnett BM. Ovarian metastases of appendiceal tumors with goblet cell carcinoidlike and signet ring cell patterns: a report of 30 cases. Am J Surg Pathol. 2007;31(10):1502-1511.

20. Taggart MW, Abraham SC, Overman MJ, Mansfield PF, Rashid A. Goblet cell carcinoid tumor, mixed goblet cell carcinoid-adenocarcinoma, and adenocarcinoma of the appendix: comparison of clinicopathologic features and prognosis. Arch Pathol Lab Med. 2015;139(6):782-790.

21. Reid MD, Basturk O, Shaib WL, Xue Y, Balci S, Choi HJ, Akkas G, et al. Adenocarcinoma ex-goblet cell carcinoid (appendiceal-type crypt cell adenocarcinoma) is a morphologically distinct entity with highly aggressive behavior and frequent association with peritoneal/intraabdominal dissemination: an analysis of 77 cases. Mod
Pathol. 2016;29(10):1243-1253.

22. Isaacson P. Crypt cell carcinoma of the appendix (socalled adenocarcinoid tumor). Am J Surg Pathol. 1981;5(3):213-224.

23. Yang C, Sun L, Zhang L, Zhou L, Zhao M, Peng Y, Niu D, et al. Diagnostic utility of SATB2 in metastatic Krukenberg tumors of the ovary: an immunohistochemical study of 70 cases with comparison to CDX2, CK7, CK20, chromogranin, and synaptophysin. Am J Surg Pathol. 2018;42(2):160-171.

24. Jiang Y, Long H, Wang W, et al. Clinicopathologic and immunohistochemical profiles of goblet cell carcinoid and typical carcinoid of the appendix. Pathol Onco Res. 2011;17(1):127-132.

25. Alsaad KO, Serra S, Schmitt A, Perren A, Chetty R. Cytokeratins 7 and 20 immunoexpression profile in goblet cell and classical carcinoids of appendix. Endocr Pathol. 2007;18(1):16-22.

26. van Eeden S, Offerhaus GJ, Hart AA, Boerrigter L, Nederlof PM, Porter E, van Velthuysen ML. Goblet cell carcinoid of the appendix: a specific type of carcinoma. Histopathology. 2007;51(6):763-773.

27. Yamagishi H, Imai Y, Okamura T, Fukuda K, Ono Y, Ban $\mathrm{S}$, Inoue $\mathrm{T}$, et al. Aberrant cytokeratin expression as a possible prognostic predictor in poorly differentiated colorectal carcinoma. J Gastroenterol Hepatol. 2013;28(12):1815-1822.

28. Terada T. An immunohistochemical study of primary signet-ring cell carcinoma of the stomach and colorectum: I. Cytokeratin profile in 42 cases. Int J Clin Exp Pathol. 2013;6(4):703-710.

29. Juez I, Rubio C, Figueras J. Multidisciplinary approach of colorectal liver metastases. Clin Transl Oncol. 2011;13(10):721-727.

30. Moorcraft SY, Ladas G, Bowcock A, Chau I. Management of resectable colorectal lung metastases. Clin Exp Metastasis. 2016;33(3):285-296.

31. Mahteme H, Sugarbaker PH. Treatment of peritoneal carcinomatosis from adenocarcinoid of appendiceal origin. Br J Surg. 2004;91(9):1168-1173.

32. Altree-Tacha D, Tyrrell J, Haas T. CDH17 is a more sensitive marker for gastric adenocarcinoma than CK20 and CDX2. Arch Pathol Lab Med. 2017;141(1):144-150.

33. Dimmler A, Geddert H, Faller G. EGFR, KRAS, BRAFmutations and microsatellite instability are absent in goblet cell carcinoids of the appendix. Pathol Res Pract. 2014;210(5):274-278.

34. Jesinghaus M, Konukiewitz B, Foersch S, Stenzinger A, Steiger K, Muckenhuber A, Gross C, et al. Appendiceal goblet cell carcinoids and adenocarcinomas ex-goblet cell carcinoid are genetically distinct from primary colorectaltype adenocarcinoma of the appendix. Mod Pathol. 2018. 